\title{
Optimal Control of Gaze Shifts
}

\author{
Andreas A. Kardamakis ${ }^{1,2}$ and Adonis K. Moschovakis ${ }^{1,2}$ \\ ${ }^{1}$ Institute of Applied and Computational Mathematics, Foundation of Research and Technology Hellas, Heraklion 71003, Crete, Greece, and ${ }^{2}$ Department of \\ Basic Sciences, Faculty of Medicine, University of Crete, Heraklion 71003, Crete, Greece
}

To explore the visible world, human beings and other primates often rely on gaze shifts. These are coordinated movements of the eyes and head characterized by stereotypical metrics and kinematics. It is possible to determine the rules that the effectors must obey to execute them rapidly and accurately and the neural commands needed to implement these rules with the help of optimal control theory. In this study, we demonstrate that head-fixed saccades and head-free gaze shifts obey a simple physical principle, "the minimum effort rule." By direct comparison with existing models of the neural control of gaze shifts, we conclude that the neural circuitry that implements the minimum effort rule is one that uses inhibitory cross talk between independent eye and head controllers.

\section{Introduction}

To execute even the simplest action, animals resort to sophisticated neural control that takes into consideration the degrees of freedom and the dynamics of the effectors used to accomplish it. The computational complexity of the processes employed to establish the relevant muscle synergies is often reduced through simplifying brain mechanisms (Bernstein, 1967). Optimal control theory (Wolpert and Ghahramani, 2000; Todorov, 2004) exploits the kinematic and dynamic stereotypes of observed movements to gain insight into these mechanisms. To this end, a performance objective is usually expressed as minimization of a "cost" function, where cost can include time, force, impulse, energy, jerk, stability, and accuracy (Nelson, 1983). In the past, jerk (Flash and Hogan, 1985), torque change (Uno et al., 1989), and variance in the presence of signal dependent noise (Harris and Wolpert, 1998) are three of the physical measures of cost that have been minimized to elucidate the principles of organization of arm reaching movements, whereas minimum-time (Clark and Stark, 1975; Enderle and Wolfe, 1987) and minimum-variance (Harris and Wolpert, 1998) have been used to predict the trajectories of rapid eye movements (saccades).

Both the difficulty of a task and the complexity of its neural control increase when two and more effectors must be coordinated. A case in point is the coordination of the eyes and the head during rapid gaze shifts. These are stereotypical movements with severely constrained metrics and kinematics. For example, the duration of head restrained saccades and head unrestrained gaze shifts depends linearly on their amplitude as does their peak velocity (Bahill et al., 1975; Tomlinson and Bahra, 1986). Also, head-free eye velocity profiles are symmetric when they accom-

Received Nov. 16, 2008; revised March 2, 2009; accepted March 30, 2009.

This study was conducted in partial fulfillment of the requirements for awarding a Ph.D. degree in the Brain and Mind Sciences to A.A.K. (cofunded by the European Social Fund and National resources). The financial support of Grant 03ED803 from the Secretariat of Research and Technology is gratefully acknowledged. We thank Dimitris Tsakiris for valuable comments.

Correspondence should be addressed to Andreas A. Kardamakis at the above address. E-mail: kardamak@edu.med.uoc.gr.

DOI:10.1523/JNEUROSCI.5518-08.2009

Copyright (C) 2009 Society for Neuroscience $\quad 0270-6474 / 09 / 297723-08 \$ 15.00 / 0$ pany small gaze shifts $\left(<20^{\circ}\right)$ but become two-peaked for larger gaze shifts (Freedman and Sparks, 1997, 2000; Roy and Cullen, 1998; Tomlinson and Bahra, 1986; Tweed et al., 1995) (for review, see Freedman, 2008). Moreover, larger gaze shifts rely on considerable head contributions, whereas smaller ones are accomplished essentially by the eyes alone while eye and head contributions to gaze shifts depend on initial eye position in the orbit (Volle and Guitton, 1993; Freedman, 2008).

The neural control of saccades and gaze shifts has attracted considerable experimental attention and several models have been proposed to account for the considerable body of evidence amassed. In the pioneering "linear summation hypothesis," Bizzi (1979) proposed that saccades and the vestibulo-ocular reflex sum linearly, such that head contributions to gaze shifts are subtracted from ocular contributions. Alternatively, the brain has been thought to use "gaze feedback control" (Laurutis and Robinson, 1986; Guitton et al., 1990). This scheme assumes that gaze (eye position in space) is the controlled variable and that a neural controller compares it to instantaneous eye position in space to create an internal representation of gaze-error, which simultaneously drives the eyes and the head until the line of sight reaches the target. Finally, more recent models of eye-head gaze shifts (Phillips et al., 1995; Freedman, 2001; Moschovakis et al., 2008), assume the existence of cross talk between their independent head- and eye-related circuits ("independent eye and head control”). Which of these models, if any, reflects reality remains a matter of debate.

In this study, we use optimal control methods to understand the functional principles of eye-head gaze shifts. We assumed that eye and head motor commands keep movement duration as short as possible while minimizing the squared sum of the magnitude of the control signals that drive the eye and head plants. This is analogous to a minimum energy dissipation criterion and we refer to it as the minimum-effort principle. This assumption suffices to predict the major kinematic features of rapid horizontal eye-head gaze shifts over a wide range of amplitudes, including: (1) realistic single-peak eye velocity profiles of head-fixed saccades as well as small and centripetal head-free gaze shifts, (2) double-peak eye velocity profiles of large centrifugal gaze shifts, 
(3) ocular components that do not exceed $35^{\circ}$ even for large gaze shifts, and (4) initial eye position dependent contributions of the eyes and the head. Minimum effort also provides insight into the interrelation between the duration and amplitude of head-fixed saccades and head-free gaze shifts. To explore possible neural mechanisms that could generate the optimal signals inferred from our model, we compared the commands generated by a "gaze feedback" model to those of an "independent eye and head" neural control model. We demonstrate that the latter can generate the commands needed to implement the herein-proposed minimal effort rule, whereas the former cannot.

\section{Materials and Methods}

We formulated the model as an open-loop optimization one that attempts to recover the best sequence of muscle activations that will minimize a performance criterion. As our performance criterion, we adopted the minimum-effort rule as a two-component cost that depends on both the eye and head control signals. To compute the optimal trajectories of the eyes and the head during head-fixed saccades and head-free gaze shifts, we minimized the time-integral of the square of the eye $\left(u_{e}\right)$ and head $\left(u_{h}\right)$ control signals (Eq. 1$)$

$$
J=\min \int_{t_{0}}^{t_{f}}\left[\alpha\left(x_{e}\right) u_{e}^{2}+\beta u_{h}^{2}\right] d t
$$

where $t_{f}-t_{0}$ is the movement duration, and $\alpha$ and $\beta$ are weights that scale the eye and head commands, respectively. The state-varying penalty $\alpha$ acts as a weight parametrizing the eye control signal. It minimizes the forces associated with the eye as it moves into eccentric positions where an increasing amount of effort is required for the agonist extraocular muscle to contract progressively more. The eye weighting function is a differentiable second-order polynomial (of the form: $\alpha=\alpha_{0}+\alpha_{1} x_{e}+$ $\alpha_{2} x_{e}^{2}$ ) that approximates extraocular muscle tension as a function of eye position (Collins, 1975; Dean, 1996). Because of this term, the eye command that controls the agonist muscle causing the eye to move into eccentric positions are penalized by the state-dependent weight function. Weight $\beta$ penalizes the head commands and is state invariant because we assume that the inertial forces are more prominent than neck muscle tension for head movements $<40^{\circ}$. In the case of very large gaze shifts $\left(>90^{\circ}\right)$, neck muscle tension and additional effectors, such as the trunk, may be incorporated into Equation 1 .

Equation 1 assumes that effort represents the control energy that is required to drive the eyes and the head and increases quadratically with the magnitude of the commands sent to these effectors. Rather than relate an optimality criterion with a physical state variable of the system, it is better to associate it with control variables by taking into account the dynamics of the mechanical components of the system (Uno et al., 1989). To account for the mechanical properties of the eye and the head, we used a second-order eye plant with time constants at 150 and $12 \mathrm{~ms}$ (van Opstal et al., 1985), and a second-order head plant with time constants at 182 and $105 \mathrm{~ms}$ (inferred from data presented in Bizzi et al., 1978). This results in a fourth-order state-space model of the gaze control system with two inputs and one output of the form, $d \mathbf{x} / d t=A \mathbf{x}+B \mathbf{u}$ and $y(t)=$ $C \mathbf{x}(t)$, which is stated below:

$$
\begin{aligned}
& \dot{x}_{1}=a_{11} x_{1}+a_{12} x_{2}+b_{1} u_{e}, \quad \dot{x}_{2}=a_{21} x_{1}, \\
& \dot{x}_{3}=a_{33} x_{3}+a_{34} x_{4}+b_{3} u_{h}, \quad \dot{x}_{4}=a_{43} x_{3}, \\
& \text { and } y=c_{2} x_{2}+c_{4} x_{4},
\end{aligned}
$$

States $x_{1}$ and $x_{2}$ in the first two state-space equations are the velocity and position states of the eye plant, respectively. Similarly, $x_{3}$ and $x_{4}$, are the velocity and position states of the head plant. The output equation $y$ represents gaze displacement, where the first term on the right-hand side is the eye position relative to the head $\left(x_{e}\right)$ and the second term is the head position with respect to space $\left(x_{h}\right)$.
Equation 1 does not include terms for online sensory feedback since experimental evidence suggests that the extraocular muscles are not endowed with a myotatic reflex (Guthrie et al., 1983) and vision is too slow to help in the control of saccades (Syka et al., 1979). To obtain the optimal control signals, we use Pontryagin's Minimum Principle (Pontryagin et al., 1962; Bryson and Ho, 1969) to derive analytical expressions for $u_{e}$ and $u_{h}$ and solve the problem as a two-point boundary value problem. According to Pontryagin's Maximum Principle, the Hamiltonian function $H$ of the system is in the form of $H(\mathbf{x}, \mathbf{u}, t)=L(\mathbf{x}, \mathbf{u}, t)+\lambda^{T}(t) f(\mathbf{x}, \mathbf{u}$, $t$ ), where function $f$ is our state-space equation (Eq. 2), and $L$ (the Lagrangian) occupies the integral part of the criterion function in Equation 1 and $\lambda$ are the set of Lagrangian multipliers (costate vectors) which correspond to each of the states, respectively. In this formulation,

$$
\begin{aligned}
H(\mathbf{x}, \mathbf{u}, t)=\alpha u_{e}^{2} & +\beta u_{h}^{2}+a_{11} x_{1} \lambda_{1} \\
& +a_{12} x_{2} \lambda_{1}+b_{1} u_{e} \lambda_{1}+a_{21} x_{1} \lambda_{2} \\
& +a_{33} x_{3} \lambda_{3}+a_{34} x_{4} \lambda_{3}+b_{3} u_{h} \lambda_{3}+a_{43} x_{3} \lambda_{4} .
\end{aligned}
$$

The optimal controls $\mathbf{u}(t)$ can be derived semianalytically by calculating the impulse response functions $\partial H / \partial \mathbf{u}$ and equating them to zero. This rule of stationarity would then result in the following expressions,

$$
u_{e}^{*}=\frac{b_{1} \lambda_{1}}{2 \alpha\left(x_{e}\right)}, \text { and } u_{h}^{*}=-\frac{b_{3} \lambda_{3}}{2 \beta} .
$$

The costate differential equations are derived by substituting Equation 4 into the Hamiltonian in Equation 3 and by applying $\dot{\lambda}(\mathrm{t})=-\partial H / \partial \mathbf{x}$,

$$
\begin{aligned}
& \dot{\lambda}_{1}=-a_{11} \lambda_{1}-a_{21} \lambda_{2}, \\
& \dot{\lambda}_{2}=-a_{12} \lambda_{1}-\left(\frac{\partial \alpha\left(x_{e}\right)}{\partial_{x_{e}}} \cdot \frac{b_{1}^{2} \lambda_{1}^{2}}{4 \alpha^{2}\left(x_{e}\right)}\right), \\
& \dot{\lambda}_{3}=-a_{33} \lambda_{3}-a_{43} \lambda_{4} \text {, and } \dot{\lambda}_{4}=-a_{34} \lambda_{3} .
\end{aligned}
$$

Boundary conditions need to be specified for $t=t_{0}$ and $t=t_{f}$ to fully define the two-point boundary value problem (refer to Fig. 1). Initial boundary conditions are given by path velocity constraints $\mathbf{x}: x_{1}\left(t_{0}\right)=$ $x_{3}\left(t_{0}\right)=0$. This implies that saccades and head movements start simultaneously with the gaze shifts they accompany as is the case in monkeys (Freedman and Sparks, 1997). Final boundary conditions are provided: (1) through $\lambda_{f}^{T}=\partial \phi / \partial \mathbf{x}$ [where $\phi=0.5\left(y-\Delta G^{\prime}\right)$, and $\Delta G^{\prime}$ is the desired gaze displacement], which is imposed on the costates $\lambda$ and lead to the following boundary conditions: $\lambda_{2}\left(t_{f}\right)=2 c_{2}\left(y-\Delta G^{\prime}\right), \lambda_{3}\left(t_{f}\right)=0$, and $\lambda_{4}\left(t_{f}\right)=2 c_{4}\left(y-\Delta G^{\prime}\right)$, and (2) $x_{1}\left(t_{f}\right)=0$, which implies that gaze shifts end together with ocular saccades as is the case in monkeys (Freedman and Sparks, 1997). Together with initial boundary conditions, the latter implies that the duration of gaze shifts equals the duration of the saccades that accompany them, as is the case in monkeys (Freedman and Sparks, 1997). After the end of the gaze shift, the head continues to move but does not alter the direction of the line of sight because of the operation of a fully functional vestibulo-ocular reflex (for a recent review, see Pelisson and Guillaume, 2009). For this reason, rather than simulate the complete trajectory of the head, we only consider the contribution of its movement until the end of the gaze shift.

Extremal trajectories are generated by simultaneously solving the sets of ordinary differential Equations 2 and 5 subject to the boundary conditions with a standard boundary-value solver. Results were obtained by using the two-point boundary value problem solver function bvp4c.m available in MatLab. The following parameters were used in the simulation of the optimal control model (Eqs. 1-5): $a_{11}=-90, a_{12}=-17.36$, $a_{21}=32, b_{1}=4, c_{2}=4.34, a_{33}=-15, a_{34}=-6.54, a_{43}=8, b_{3}=4, c_{4}$ $=2.81, \alpha_{0}=9.1, \alpha_{1}=0.36, \alpha_{2}=0.014$ and $\beta=18$. The first five parameters arise from the conversion of the transfer function of the ocular plant into its state-space representations and correspond to time constants equal to 150 and $12 \mathrm{~ms}$ (van Opstal et al., 1985). Similarly, the next five parameters apply to the same conversion for the head plant and correspond time constants equal to 182 and $105 \mathrm{~ms}$, which, as described above, are inferred from the biomechanics data of Bizzi et al. (1978). The 
a. Time course of Movement Cost

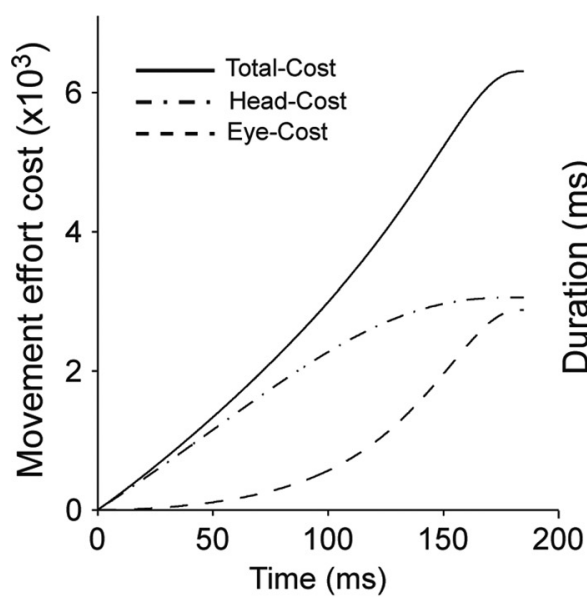

b. Head-fixed saccades

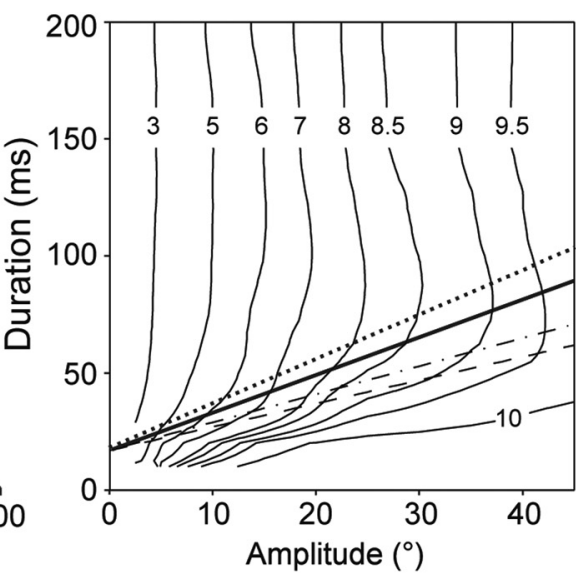

c. Head-free gaze shifts

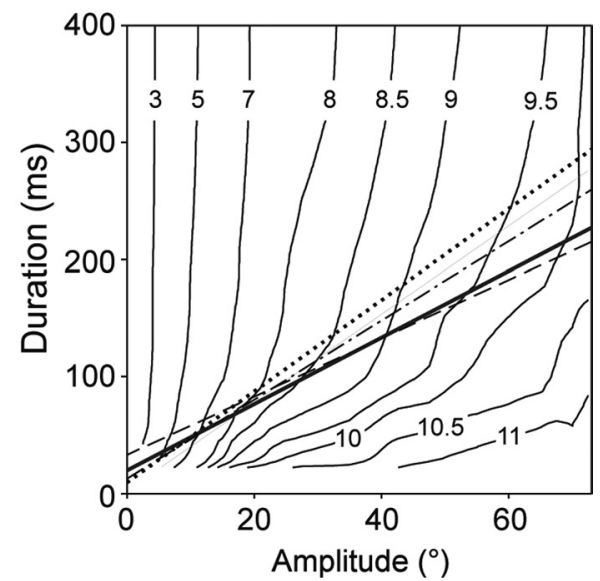

Figure 1. Effort and movement duration. $a$, Time course of effort for a medium-sized $\left(50^{\circ}\right)$ gaze shift. Solid line, Combined effort for both eye and head. Dashed line, Effort associated with eye component. Dashed-dot line, Effort associated with head component. Note that the total effort of the movement is the cost value of the combined effort at the end of the movement. $\boldsymbol{b}$, Iso-effort contours for head-fixed saccades. Abscissa, Saccade amplitude; ordinate, saccade duration. Lines indicate the amplitude- duration relationship obtained from Fuchs (1967), King et al. (1986), van Gisbergen et al (1981), and the one used in our optimal control model (dashed line, dotted line, dashed-dot line and solid line, respectively). c, Iso-effort contours for head-free gaze shifts. Abscissa, Amplitude; ordinate, duration. Lines indicate the amplitude-duration relationship obtained from Tomlinson and Bahra (1986), Phillips et al. (1995), Freedman et al. (1997), and the one used in our simulations of eye-head gaze shifts (dashed line, dotted line, dashed-dot line and solid line, respectively). Numbers indicate the total effort required to accomplish the movement (on the log-scale).

remaining four parameters are associated with the eye and head control energy penalties. The first three $\left(\alpha_{0}, \alpha_{1}, \alpha_{2}\right)$ were obtained from iterative curve-fitting of the extraocular muscle length-tension curve of Collins (1975) and are approximately similar to the parameters estimated by Dean (1996). Our simulations are not sensitive to the precise value of parameter $\beta$ (which penalizes the head control energy), provided that $\beta>\alpha_{0}$, unless very large values of $\beta$ are reached, in which case shifts of the line of sight are accomplished by the eyes alone.

\section{Results}

The generation of saccades and eye-head gaze shifts is governed by an optimal balance between "effort" and movement duration. We define effort as the time-integral of the squared sum of the motor commands driving the relevant plants (see Eq. 1 in Materials and Methods) that represents the control energy required to efficiently displace an effector from one point in space to another. This movement effort cost is plotted as a function of time in Figure $1 a$ (solid) for a medium-sized $\left(50^{\circ}\right.$ in amplitude) centrifugal gaze shift. As shown here, the effort needed to execute the ocular component of such a gaze shift (dashed) grows parabolically as the eye moves into more eccentric locations and extraocular muscle tension increases quadratically (Collins, 1975; Dean, 1996). However, the initial part of the curve describing the cost of the head component (dash-dots) is linear and grows to an asymptote toward the end of the gaze shift as it enters into its deceleration phase.

To understand the interrelation between movement duration, amplitude, and effort, these three quantities are simultaneously displayed as iso-effort contour plots for head-fixed eye saccades in Figure $1 b$. Each contour line represents a constant level of effort for different combinations of amplitude and duration. For every movement amplitude, effort becomes almost movementsize invariant beyond a critical duration, coincident with the inflection points of the iso-effort contours shown in Figure $1 b$. Further decrease in duration beyond this critical value requires additional effort that increases exponentially in inverse proportion to duration. One would thus expect the amplitude-duration relationship of primate saccades not to venture far from these inflection points. An amplitude-duration relationship shallower than this would describe saccades that last less but at the cost of additional effort, whereas a steeper one would characterize loweffort saccades that take a long time to complete. The amplitudeduration relationship of experimental subjects often passes through or very near these optimal points. For example, such a relationship (1.2 ms/deg) (Fig. $1 b$, dashed-dots) is obeyed by the two monkeys of van Gisbergen et al. (1981) and one of the monkeys (\#1) of King et al. (1986). Yet, the amplitude-duration relationships can differ a lot between subjects. Slopes as high as 1.9 $\mathrm{ms} /$ deg (Fig. 1 $b$, dotted line) were obtained from monkey \#2 of King et al. (1986), whereas slopes as low as $1 \mathrm{~ms} / \mathrm{deg}$ (Fig. $1 b$, dashes) were found by Fuchs (1967). However, even in these somewhat extreme cases, relationships experimentally determined from monkeys do not depart much from the hereindetermined optimal one. To simulate optimal head-fixed saccades, a value of $1.6 \mathrm{~ms} / \mathrm{deg}$ (Fig. $1 b$, solid line) is used, which leaves the amplitude-duration relationship comfortably within the range of experimentally determined ones.

Similarly, Figure $1 c$ displays the iso-effort contours for headfree gaze shifts. Their inflection points are not as easily discernible and span a bigger region than those of the iso-effort contour plots of saccades and effort becomes invariant at much higher duration values. Experimentally determined gaze amplitude-duration relationships also intersect the iso-effort contours when the latter become size invariant. The dashed line of Figure $1 c$ illustrates a case in point documented by Tomlinson and Bahra (1986) and characterized by a slope of $2.4 \mathrm{~ms} / \mathrm{deg}$. Other published gaze amplitude-duration relationships are even steeper. For example, a slope equal to $3.4 \mathrm{~ms} / \mathrm{deg}$ (Fig. 1c, dashed-dot) was found in monkey T by Freedman and Sparks (1997) and a slope equal to $3.7 \mathrm{~ms} / \mathrm{deg}$ (Fig. 1c, dotted line) was obtained from monkey (RO) by Phillips et al. (1995). A value of $2.85 \mathrm{~ms} / \mathrm{deg}$ (Fig. 1c, solid line) is used to simulate optimal eye-head gaze shifts.

If the minimum effort principle holds, it should simultaneously apply to both saccade and gaze shift kinematics. To explore whether this is the case, we first simulated a series of headfixed saccades. Figure $2 a$ shows the velocity profiles of five saccades ranging from $5^{\circ}$ to $40^{\circ}$ which obey the amplitude-du- 
ration relationship that follows the solid line of Figure $1 b$. The velocity profiles of saccades smaller than $20^{\circ}$ are symmetrical, whereas larger saccades exhibit a short acceleration phase followed by a longer deceleration phase. Consistent with experimental observations in monkeys (Freedman, 2008) and humans (van Opstal and van Gisbergen, 1987), the skewness of the velocity profiles increases with movement size. This is shown in the inset of Figure $2 a$ which illustrates the velocity profiles of three typical saccades from the monkey [extracted from the study by Freedman (2008), their Fig. 2b]. Although the onset and offset of our simulated saccades is more abrupt than those of primates, this is attributable to our choice of a second-order ocular plant rather than a third-order one to model eye movements [compare Fig. $2 a$ of the present study to the study by Harris and Wolpert (1998), their Fig. 2b,c]. Also consistent with experimental observations (Collewijn et al., 1988, Fuchs, 1967), the peak values reached by our optimal velocity profiles display a soft saturation. Rather than having to explicitly constrain a model to reproduce them, several qualitative features of saccadic velocity profiles thus emerge from the application of the minimum effort rule.

After showing that the minimum effort rule provides an accurate account of saccade kinematics we explored whether it also captures the main features of eye-head gaze shifts. To this end, we simulated a series of horizontal gaze shifts with amplitudes ranging from $5^{\circ}$ to $75^{\circ}$ and obeying an amplitude-duration relationship such as the one shown in Figure $1 c$. No constraints were imposed on the sizes of the individual eye and head components of the gaze shifts. Instead their sum is constrained to equal retinal error $\left(\Delta G^{\prime}\right)$ for all gaze shifts spanning the entire range that was simulated (Fig. $3 c$ ). Figure $3 b$ shows the relation between the size of the simulated gaze shift and the amplitude of the contribution of the head (open diamonds) plotted together with empirical data obtained from monkey T of Freedman and Sparks (1997) and illustrated in their Figure 6C (dots). As in experimental subjects (Freedman and Sparks, 1997; Stahl, 1999), small (15-20) simulated gaze shifts are accomplished by the eyes virtually alone, and the contribution of the head remains negligible (Fig. $3 b$ ). As is the case in monkeys [Fig. $3 a$, dots: extracted from the study by Freedman and Sparks (1997), their Fig. 6E], the ocular components of simulated gaze shifts (Fig. $3 a$, open diamonds) do not exceed $30-35^{\circ}$ even for large gaze shifts. It should be pointed out that both the saturation of the ocular contribution for large gaze shifts and the negligible contribution of the head for small gaze shifts are not caused by the imposition of additional constraints, such as saturating and threshold nonlinearities, but are instead emergent properties of the minimum effort principle.

The assumption that it underlies eye-head coordination also allowed us to reproduce the eye velocity profiles of gaze shifts widely ranging in size. Figure $2 b$ shows three examples, the small- est one from a $20^{\circ}$ movement, a medium-sized one from a $40^{\circ}$ movement, and the largest one from a $70^{\circ}$ gaze shift. As the size of the gaze shift increases, peak velocity decreases and duration increases. Furthermore, the shape of the velocity profiles changes. It is almost symmetrical for the small one, skewed in the case of the medium-sized one, and dual-peaked for the biggest of the three. They resemble the eye velocity traces extracted from small, medium, and large, respectively [Freedman and Sparks (1997), their Fig. $8 B$, traces a-c], head-free movements of the monkey shown in the inset of Figure $2 b$. In both the monkey and the model, it would thus appear as if the eye must accelerate, subsequently decelerate, and then reaccelerate whenever head-free gaze shifts are large enough, i.e., when they are accompanied by large head movements. Indeed, such twin peak eye velocity profiles have been repeatedly documented (Tomlinson and Bahra, 1986; Tweed et al., 1995; Freedman and Sparks, 1997, 2000; Roy and Cullen, 1998) for head-free gaze shifts of monkeys accompanied by large head contributions. To obtain these velocity profiles, we used the amplitude-duration relationship shown as solid line in Figure $1 c$, as a boundary condition in the cost function we minimized. Use of a shallower amplitude-duration relationship [for example, one that lies below the line in Fig. $1 c$ obtained by Tomlinson and Bahra (1986)] leads to movements of shorter duration that are also characterized by the disappearance of the second peak in their velocity profiles.

The simulated movements we examined so far were assumed 

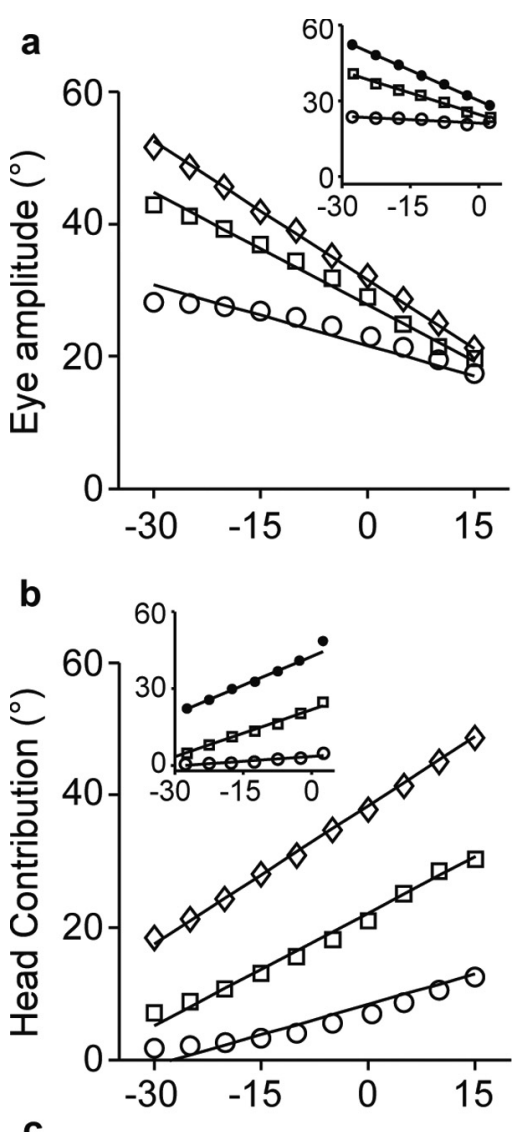

C

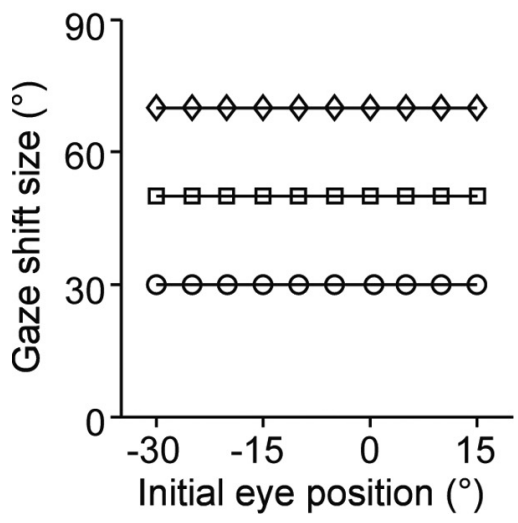

Figure 4. Metrics of gaze shifts with the eyes starting from different initial positions. $\boldsymbol{a}-\boldsymbol{c}$, Size of eye $(\boldsymbol{a})$ and head $(\boldsymbol{b})$ contributions to rightward head-free gaze shifts (c) of constant amplitudes equal to $30^{\circ}$ (open circles), $50^{\circ}$ (open squares), and $70^{\circ}$ (open diamonds), as a function of initial eye position (abscissa). Negative values indicate leftward initial eye positions. Data were fit with least-squares regression lines the slopes of which were the following: (a) $30^{\circ}$ : $-0.3 ; 50^{\circ}:-0.5 ; 70^{\circ}$ : -0.7 and $(\boldsymbol{b}) 30^{\circ}: 0.3 ; 50^{\circ}: 0.5 ; 70^{\circ}: 0.7$. Insets, Data extracted from Figure 15B (in $\boldsymbol{b}$ ) and Figure 15C (in $\boldsymbol{a}$ ) of the study by Freedman and Sparks (1997).

to start from straight ahead initial position of the eyes. Changing this initial condition allowed us to explore the interaction between eye and head contributions to gaze shifts. These are known to depend on the position of the eyes in the orbit at the onset of the gaze shift (Freedman and Sparks, 1997). To investigate the origins of this phenomenon, three gaze shift sizes, $30^{\circ}, 50^{\circ}$, and $70^{\circ}$ were simulated (Fig. 4), whereas initial eye position varied between $30^{\circ}$ to the left and $15^{\circ}$ to the right of straight ahead. Although this was not imposed on our optimal control model through the adoption of any additional constraints, as shown in Figure $4 a$, the size of the ocular contributions to the gaze shift is inversely correlated to initial eye position. To generate constant amplitude gaze shifts (Fig. 4c), the head contribution must exhibit the opposite relation (Fig. $4 b$ ), i.e., a positive slope with respect to initial eye position. A second emergent property of the minimum effort rule associated with position sensitivity of gaze shifts is the fact that the larger the size of the gaze shift, the steeper the regression slope. The same is true of the ocular and the head contributions to head-free gaze shifts of the monkey as shown in the insets of Figure 4, $a$ and $b$, respectively [which includes data extracted from the study by Freedman and Sparks (1997), their Fig. $15 B, C$, respectively].

\section{Discussion}

We used optimal control theory to disclose a principle of organization of eye-head gaze shifts, the minimum effort rule. Iso-effort contours reveal that the optimal performance of head-fixed saccades and head-free gaze shifts take advantage of a balance between effort and time. Every muscular system is subject to fatigue when used continuously and this is particularly true of the ocular muscles of primates and humans, which are known to shift their gaze more than $10^{5}$ times a day (Bahill and Stark, 1975). Once fatigued, saccades become slower with lower peak velocities that violate the main sequence relationship (Bahill and Stark, 1975). To reduce the risk of fatigue, the saccadic system could limit its operation to between 5 and 9.5 on the effort scale (Fig. 1), thus avoiding the use of excessive forces. This could be accomplished by increasing movement duration. However, movements of the eyes compromise vision and thus their duration should preferably be kept to a minimum. One would thus intuitively expect optimal gaze shifts from the trade-off between effort and movement duration. As suggested before, extraocular muscles and the neurons that control them are endowed "with one or more safety factors, which ensure accurate and consistent performance regardless of the demands placed on them" (Fuchs and Binder, 1983). Movement duration could be one such safety factor. In turn, eye and head contributions to gaze shifts are constrained by a trade-off between the forces associated with the high inertia of head and the viscoelastic forces associated with rapid eye movements. Such a trade-off is reached at a point determined from the two weights ( $\alpha$ and $\beta$ ) in the equation that describes the functionality of the system (Eq. 1) and penalize the size of the motor command sent to the eye $(\alpha)$ and to the head $(\beta)$. Evidently one could generalize this argument to encompass additional effectors, such as the trunk and the lower body.

An inspection of optimal eye and gaze trajectories demonstrates their similarity to those executed by animals. First, the velocity profiles of small head-fixed saccades $\left(<20^{\circ}\right)$ are symmetrical, whereas those of bigger movements are skewed with short acceleration and longer deceleration phases (Fig. 2), as in humans (Collewijn et al., 1988, van Opstal and van Gisbergen, 1987) and monkeys (Freedman, 2008). Also, consistent with experimental observations (Freedman and Sparks, 1997; Syka et al., 1979), smaller simulated gaze shifts $\left(<20^{\circ}\right)$ are not accompanied by head movements and ocular components do not exceed $30-35^{\circ}$ even for large $\left(40-90^{\circ}\right)$ gaze shifts (Fig. 3). Moreover, as in natural movements (Freedman and Sparks, 1997), the eye velocity profiles of relatively small head-free gaze shifts $\left(<15^{\circ}\right)$ are unimodal and rather symmetrical, whereas larger ones are characterized by lower peak values and two-humped velocity profiles (Tomlinson and Bahra, 1986; Tweed et al., 1995; Freedman and Sparks, 1997, 2000; Roy and Cullen, 1998). Furthermore, the size of the contributions of the eye and the head depend on the initial orbital position of the eyes (Volle and Guitton, 1993; Freedman 
and Sparks, 1997). The head contributes progressively less (and the eyes more) to gaze shifts of the same size as initial eye position is directed contralaterally to the impending gaze shift. The opposite is true when the eyes are directed ipsilaterally. The eye position dependence increases with the size of the gaze shift. Comparison of Figure 4 of this study to the study by Freedman and Sparks (1997), their Figure 15 , demonstrates that our model reproduces quite well the overall features of this effect as determined experimentally for gaze shifts of 30,50 , and $70^{\circ}$. For example, the slopes of the regression lines relating head amplitude to initial eye position increased from 0.3 to 0.7 as the size of the simulated gaze shift increased from 30 to $70^{\circ}$. The same is true for the relationship between ocular contributions and initial eye position; for example, the slope $(-0.7)$ we obtained for large gaze shifts $\left(70^{\circ}\right)$ lies in between the values determined experimentally $(-0.43$ and -0.85$)$ in two monkeys executing $70^{\circ}$ gaze shifts (Freedman and Sparks, 1997).

Inspection of the optimal eye-head motor commands provides some insight into the signals driving the eyes and the head. Figure $5 a$ illustrates the dynamic portion of the control signals leading to head-free gaze shifts of three different sizes starting from straight ahead. These motor commands are velocity-dependent and compensate for inertial effects, and consistent with the separation principle (Guigon et al., 2007) they can be optimized independently from the static components reflected in the tonic activity compensating for gravitational and elastic restoring forces. The commands sent to the eye are tightly linked to eye velocity, resulting from the fact that the eye is virtually inertia-less and is dominated by viscous elements (Robinson, 1981). However, the head is dominated by its high inertial component and thus the agonist commands sent to it are tightly linked to the acceleration phase of its movement (Zangemeister et al., 1981). This is followed by antagonist commands applied by the opposing muscle to decelerate the head movement (Hannaford et al., 1986). Our model demonstrates that for a certain amount of effort, the size of head contributions to gaze shifts increases when their acceleration phase coincides with the movement of the eye. This is resulting from the fact that signals conveyed to antagonist neck muscles (by convention these are considered to have a negative sign) induce the expenditure of effort (because of the quadratic form of the minimum effort performance criterion) and decelerate the head rather than assist agonist muscles in pushing it further in the same direction. Thus the present model provides an intuitive explanation for the fact that the duration of the ocular component of gaze shifts coincides with the acceleration phase of their head components (Guitton et al., 1990).
Optimal Control using Minimum Effort rule

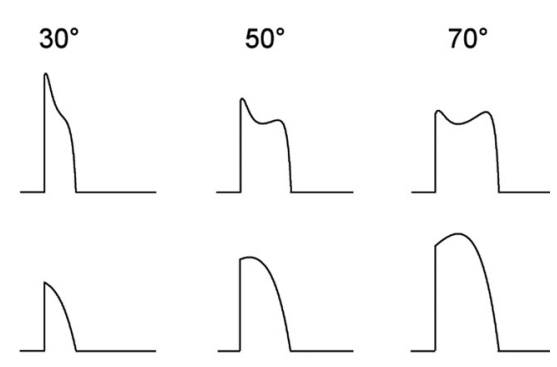

Independent Eye-Head Control: Crosstalk

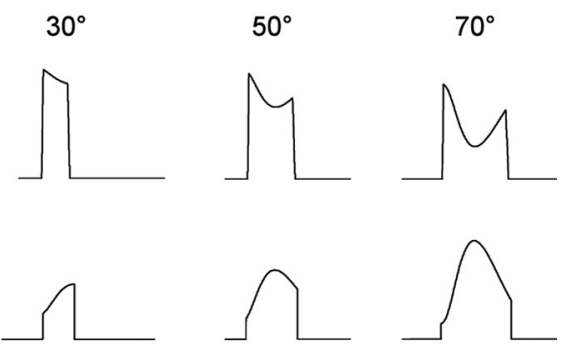

Gaze Feedback Control

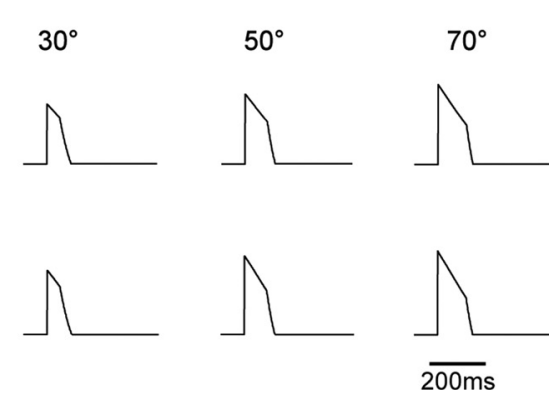

Figure 5. Motor commands controlling gaze shifts $(\Delta G)$ of 30,50 , and $70^{\circ}$. $a$, Signals derived from the minimum effort rule. $\boldsymbol{b}$, Left, Schematic illustrating the major building blocks of a neural model that assumes independent eye and head control and (right). Negative signs next to the arrowheads indicate inhibitory connections. Allother neural models $(\boldsymbol{b})$ and $(\boldsymbol{c})$ have been implemented in Simulink of the Matlab environment. Time bar (200 ms) applies to all waveforms. The amplitude of the motoneuronal eye and head units (measured in spikes per second) vary because of differences among the gains of their corresponding eye and head plants.

As shown in Figure $5 a$, the signal controlling the head need not reach its peak value together with the signal controlling the eye. As the amplitude and duration of signals controlling the head increase, the shape of the optimal eye commands change from single-peaked to two-humped profiles. Because of their rapid responsiveness, the velocity of the eyes can follow much faster control signals, and distinct peaks can occur near the beginning and toward the end of large gaze shifts. However, the head is a slower plant (Bizzi et al., 1978) and slower signals suffice to drive it through realistic trajectories. The command signals sent to it display a single peak, which often occurs approximately halfway through the activation of the agonist muscle. The aforementioned details concerning the waveforms of the optimal signals driving the eye and the head apply to gaze shifts of all sizes and emerge from the minimum effort rule.

What alternative optimality principles could govern gaze shifts? It has been argued that saccades are programmed such that 
their duration is kept to a minimum (Clark and Stark, 1975; Enderle and Wolfe, 1987). Assuming that the saccadic system is linear, the minimum-time requirement implies that it uses "bang-bang" control, i.e., that its output is at its maximum force limits (positive or negative) over the entire movement. As argued before (Harris and Wolpert, 1998), adoption of the minimum time rule does not lead to realistic saccade velocity profiles. Moreover, minimizing movement time leads one to expect larger contributions from the faster of the two effectors (the eyes) during coordinated eye-head gaze shifts. In fact, ocular contributions are smaller than expected from the oculomotor range since the eyes are known to reach eccentricities not exceeding 30-35 (Freedman and Sparks, 1997), even when monkeys execute large eyehead gaze shifts and although their oculomotor range is $45-50^{\circ}$. Minimum jerk (Flash and Hogan, 1985) or torque change (Uno et al., 1989) models have been proposed for arm movements, but are unlikely to provide the underlying principle of saccades and eye-head gaze shifts because they fail to account for important kinematic features of eye movements, such as their skewed and dual peak velocity profiles (Harris and Wolpert, 1998). Finally, minimum variance in the presence of signal-dependent noise has been invoked to account for saccade trajectories (Harris and Wolpert, 1998). Indeed, such a model could account for some of the psychophysics explained by our model such as the fact that the ocular components do not exceed some value ( $\sim 30$ degrees in the monkey). However, it is not obvious to us that it would account for the twin-peak eye-velocity profiles of large enough gaze shifts or for the initial eye position sensitivity of eye and head contributions to gaze shifts.

Our optimal control model describes eye-head coordination at the computational level and need not be relevant to the premotor circuits responsible for saccades and gaze shifts. The principles of operation of the system gleaned from such models are independent of brain structures and neural processes. It is, however, meaningful to ask what neural circuitry could generate motor commands consistent with those derived from the minimum effort rule. To answer this question, optimal control approaches must be supplemented with models of the neural control of gaze shifts that rely on systems theory to link neurophysiology (and neuroanatomy) to psychophysics (and neurology since the "lesion" of model units can be readily simulated).

Control systems theoretic approaches have been widely used to model the information flow and signal transformations in premotor circuitry controlling saccades and gaze shifts (Moschovakis et al., 1996). Models of the neural control of gaze shifts can be classified into two categories: (1) gaze feedback control and (2) independent eye and head control. These two classes of models differ in the control strategy they employ to accurately shift the line of sight. Gaze feedback models (Fig. $5 c$ ) are extensions of the eye position saccadic controller (van Gisbergen et al., 1981) and assume that desired gaze position is compared with current gaze position, so that the resulting gaze-position-error signal drives both the eyes and the head (Laurutis and Robinson, 1986; Guitton and Volle, 1987; Guitton et al., 1990). In contrast, independent eye and head control models (Fig. 5b) assume that the SC command is decomposed into separate eye and head components driving their respective plants, independently. These models use an inhibitory interaction between the head portion and the eye portion of their circuitry, mediated by the VOR (Bizzi, 1979; Whittington et al., 1984) or an efference copy of the head command (Phillips et al., 1995; Freedman, 2001; Moschovakis et al., 2008).

To examine which gaze control model generates commands best resembling those inferred from the present study, we simulated an independent eye and head control model (Fig. 5b) and a gaze feedback control model (Fig. $5 c$ ). We decided to simulate the model of Moschovakis et al. (2008) as representative of the independent control models and that of Guitton et al. (1990) as representative of the gaze feedback control models because there are parameter specifications allowing the numerical simulation of both models. The right-hand side of Figure $5, b$ and $c$, shows the phasic component of the commands sent to the eye (top rows) and the agonist neck muscle (bottom rows) obtained from the independent control (Fig. 5b) and gaze feedback model (Fig. 5c), respectively, for a 30,50 , and $70^{\circ}$ gaze shift. As movement amplitude increases, the peak value of the commands sent to the ocular plant of the independent control model decreases and they display a second peak toward the end of large gaze shifts (Fig. 5b), as do the waveforms produced by the minimum effort principle (Fig. 5a, top row) and the velocity profiles of primate head free eye movements (Tomlinson and Bahra, 1986; Tweed et al., 1995; Freedman and Sparks, 1997, 2000; Roy and Cullen, 1998). Clearly, such signals are not produced by the gaze feedback model (Fig. 5c). For example, unlike those required to optimally drive the eyes (Fig. 5a), the amplitude of the signals sent by the gaze feedback model to the eye and the head resemble each other, and simply scale with movement size while they do not display any distinct peaks.

We demonstrated that the minimum effort rule is an important design principle of eye-head motor coordination and that the CNS could implement it through inhibitory cross talk between independent eye and head controllers. We also demonstrated that such an independent control strategy can generate motoneuronal discharges matching the commands inferred from our optimal control model. Thus, results from our optimal control and neural systems modeling converge toward a unified framework of eye-head motor coordination. We anticipate that the integration of optimal control and system modeling of neural processes will prove decisive in efforts to understand the neural control of action.

\section{References}

Bahill AT, Stark L (1975) Overlapping saccades and glissades are produced by fatigue in the saccadic eye movement system. Exp Neurol 48:95-106.

Bahill AT, Clark MR, Stark L (1975) Dynamic overshoot in saccadic eye movements is caused by neurological control signal reversals. Exp Neurol 48:107-122.

Bernstein N (1967) The coordination and regulation of movements. New York: Pergamon.

Bizzi E (1979) Strategies of eye-head coordination. Prog Brain Res 50:795-803.

Bizzi E, Dev P, Morasso P, Polit A (1978) Effect of load disturbances during centrally initiated movements. J Neurophysiol 41:542-556.

Bryson A, Ho Y (1969) Applied optimal control. Waltham, MA: Blaisdell.

Clark MR, Stark L (1975) Time optimal behavior of human saccadic eye movement. IEEE Trans Automat Control 20:345-348.

Collewijn H, Erkelens CJ, Steinman RM (1988) Binocular coordination of human horizontal saccadic eye-movements. J Physiol 404:157-182.

Collins CC (1975) The human oculomotor control system. In: basic mechanisms of ocular motility and their clinical implications (Lennerstrand G, y Rita PB, eds), pp. 145-180. Oxford: Pergamon.

Dean P (1996) Motor unit recruitment in a distributed model of extraocular muscle. J Neurophysiol 76:727-742.

Enderle JD, Wolfe JW (1987) Time-optimal control of saccadic eye movements. IEEE Trans Biomed Eng 34:43-55.

Flash T, Hogan N (1985) The coordination of arm movements: an experimentally confirmed mathematical model. J Neurosci 5:1688-1703.

Freedman EG (2001) Interactions between eye and head control signals can account for movement kinematics. Biol Cybern 84:453-462. 
Freedman EG (2008) Coordination of the eyes and head during visual orienting. Exp Brain Res 190:369-387.

Freedman EG, Sparks DL (1997) Eye-head coordination during head-unrestrained gaze shifts in rhesus monkeys. J Neurophysiol 77:2328-2348.

Freedman EG, Sparks DL (2000) Coordination of the eyes and head: movement kinematics. Exp Brain Res 131:22-32.

Fuchs AF (1967) Saccadic and smooth pursuit eye movements in the monkey. J Physiol 191:609-631.

Fuchs AF, Binder MD (1983) Fatigue resistance of human extraocular muscles. J Neurophysiol 49:28-34.

Guigon E, Baraduc P, Desmurget M (2007) Computational motor control: Redundancy and invariance. J Neurophysiol 97:331-347.

Guitton D, Volle M (1987) Gaze control in humans: eye-head coordination during orienting movements to targets within and beyond the oculomotor range. J Neurophysiol 58:427-459.

Guitton D, Munoz DP, Galiana HL (1990) Gaze control in the cat: studies and modeling of the coupling between orienting eye and head movements in different behavioral tasks. J Neurophysiol 64:509-531.

Guthrie BL, Porter JD, Sparks DL (1983) Corollary discharge provides accurate eye position information to the oculomotor system. Science 221:1193-1195.

Hannaford B, Kim WS, Lee SH, Stark L (1986) Neurological control of head movements: inverse modelling and electromyographic evidence. Math Biosci 78:159-178.

Harris CM, Wolpert DM (1998) Signal-dependent noise determines motor planning. Nature 394:780-784.

King WM, Lisberger SG, Fuchs AF (1986) Oblique saccadic eye movements of primates. J Neurophysiol 56:769-784.

Laurutis VP, Robinson DA (1986) The vestibulo-ocular reflex during human saccadic eye movements. J Physiol 373:209-233.

Moschovakis AK, Scudder CA, Highstein SM (1996) The microscopic anatomy and physiology of the mammalian saccadic system. Prog Neurobiol 50:133-254

Moschovakis AK, Kardamakis AA, Grantyn A (2008) A new model of primate eye-head gaze shifts. Soc Neurosci Abstr 34.263.1.

Nelson WL (1983) Physical principles for economies of skilled movements. Biol Cybern 46:135-147.

Pelisson D, Guillaume A (2009) Eye-head coordination. In: Encyclopedia of neuroscience, Vol. 2 (Binder MD, Hirokawa N, Windhorst U, eds), pp. 1545-1548. Berlin: Springer.
Phillips JO, Ling L, Fuchs AF, Siebold C, Plorde JJ (1995) Rapid horizontal gaze movement in the monkey. J Neurophysiol 73:1632-1652.

Pontryagin LS, Boltyanskii VG, Gamkrelidze RV, Mischenko EF (1962) The mathematical theory of optimal processes. New York: Wiley.

Robinson DA (1981) Control of eye movements. In: Handbook of physiology. The nervous system, Vol. 2 (Brooks V, ed), pp. 1275-1320. Baltimore: Williams and Wilkins.

Roy JE, Cullen KE (1998) A neural correlate for vestibulo-ocular reflex suppression during voluntary eye-head gaze shifts. Nat Neurosci 1:404-410.

Stahl JS (1999) Amplitude of human head movements associated with horizontal saccades. Exp Brain Res 126:41-54.

Syka J, Popelár J, Boźkov V (1979) Responses of neurons in the superior colliculus of the cat to stationary and moving visual stimuli. Vision Res 19:213-219.

Todorov E (2004) Optimality principles in sensorimotor control. Nat Neurosci 7:907-915.

Tomlinson RD, Bahra PS (1986) Combined eye-head gaze shifts in the primate. I. Metrics. J Neurophysiol 56:1542-1557.

Tweed D, Glenn B, Vilis T (1995) Eye-head coordination during large gaze shifts. J Neurophysiol 73:766-779.

Uno Y, Kawato M, Suzuki R (1989) Formation and control of optimal trajectory in human multijoint arm movement: minimum torque-change model. Biol Cybern 61:89-101.

van Gisbergen JAM, Robinson DA, Gielen S (1981) A quantitative analysis of generation of sacccadic eye movements by burst neurons. J Neurophysiol 45:417-442.

van Opstal AJ, van Gisbergen JA (1987) Skewness of saccadic velocity profles: a unifying parameter for normal and slow saccades. Vision Res 27:731-745.

van Opstal AJ, van Gisbergen JAM, Eggermont J (1985) Reconstruction of neural control signals for saccades based on an inverse method. Vision Res 25:789-801.

Volle M, Guitton D (1993) Human gaze shifts in which head and eyes are not initially aligned. Exp Brain Res 94:463-470.

Whittington DA, Lestienne F, Bizzi E (1984) Behavior of preoculomotor burst neurons during eye-head coordination. Exp Brain Res 55:215-222.

Wolpert DM, Ghahramani Z (2000) Computational principles of movement neuroscience. Nat Neurosci 3:1212-1217.

Zangemeister WH, Jones A, Stark L (1981) Dynamics of head movements trajectories: Main sequence relationship. Exp Neurol 71:76-91. 